\title{
Gender differences in neuropsychological functioning of New Zealand adolescents with and without Attention Deficit Hyperactivity Disorder
}

\author{
Julia J. Rucklidge * \\ University of Canterbury, New Zealand
}

\begin{abstract}
Only recently have studies included a female Attention Deficit Hyperactivity Disorder (ADHD) sample when investigating neurocognitive functioning of individuals with ADHD. As such, the generalizability of findings of impaired executive functioning is limited to ADHD males. This study compared four groups: 30 male controls, 35 female controls, 24 males with ADHD, and 25 females with ADHD, aged 13 to 17 years. Participants were assessed using the K-SADS-PL and Conners' Rating Scales and completed tests of rapid naming, processing speed, memory, inhibition, set-shifting, and interference. Results showed that the males with ADHD and females with ADHD performed similarly with only one notable difference: males with ADHD showed some evidence of more impaired inhibition than females with ADHD. In contrast, after controlling for reading ability, comorbidity, and IQ, both males and females with ADHD showed some impairment in working memory, naming speeds, processing abilities, and inhibitory deficits as compared to controls. This study supports the growing literature documenting impaired neurocognitive functioning in both males and females with ADHD.
\end{abstract}

Key words: Attention Deficit Hyperactivity Disorder (ADHD); Gender differences; Neurocognitive functioning; Neuropsychological functioning; Reading Disability (RD); Sex differences

\section{Introduction}

Over the last decade, there has been a steady growth in studies investigating gender differences in Attention Deficit Hyperactivity Disorder (ADHD), a disorder characterised by

\footnotetext{
* Department of Psychology, University of Canterbury, Private Bag 4800, Christchurch, New Zealand. Email: Julia.rucklidge@canterbury.ac.nz
} 


\section{J. Rucklidge}

attention problems, impulsivity and overactivity (American Psychiatric Association, 2000). Given the insurmountable evidence showing deficits in cognitive and executive functioning (Tannock, 1998), behavioural inhibition (Barkley, 1997; Schulz et al., 2004; Slaats-Willemse, Swaab-Barneveld, De Sonneville, Van Der Meulen, \& Buitelaar, 2003), motor control (Carte, Nigg, \& Hinshaw, 1996), processing and naming speed (Tannock, Martinussen, \& Fritjers, 2000), working memory (Martinussen, Hayden, Hogg-Johnson, \& Tannock, 2005; West, Houghton, Douglas, \& Whiting, 2002), and estimation of time (Toplak, Rucklidge, Hetherington, John, \& Tannock, 2003) in mainly male ADHD samples, there has been a recent focus to determine whether these well documented problems in males with ADHD extend to females with ADHD.

While earlier studies suggested that females with ADHD may be less impaired than males with ADHD in tests of cognitive functioning (Seidman et al., 1997), evidence has been building that females are likely as impaired as males with ADHD, and in some cases, more impaired. Consistent with an earlier meta-analytic review (Gaub \& Carlson, 1997), a recent meta-analysis concluded that while ADHD females show poorer intellectual functioning than ADHD males (Gershon, 2002), no other differences in cognitive functioning were noted. Arcia and Conners (1998) did not find any gender differences in ADHD on tests of inhibition, spatial memory and planning, and overall cognitive abilities. While Rucklidge and Tannock (2002) failed to document any gender differences in an ADHD sample on tests of naming speed, working memory, and inhibition they found males with ADHD were slower with processing information than females with ADHD. A recent study by Seidman and colleagues (2005) found that across two developmental age groups (preteen and teenage years), their sample of ADHD girls and boys diagnosed using DSM-III-R showed similar levels of executive dysfunction (no gender differences) across a broad range of abilities including memory, naming speed, planning and set-shifting, and behavioural inhibition. This overall 
lack of gender differences in cognitive functioning has also been documented in an adult sample (Biederman, Faraone, Monuteaux, Bober, \& Cadogen, 2004).

Our knowledge of the functioning of ADHD females as compared with Control girls has also been growing. Similar to the evidence of broad deficits in neuropsychological functioning of males with ADHD, research has indicated the pattern is likely to be similar in females. For example, females with ADHD have been shown to perform more poorly on tests of cognitive functioning (Rucklidge \& Tannock, 2001), working memory and processing speed (Hinshaw, 2002), driving abilities (Nada-Raja et al., 1997), executive function oculomotor functioning (Castellanos et al., 2000), and set-shifting, planning, vigilance, and inhibitory control (Hinshaw, Carte, Sami, Treuting, \& Zupan, 2002; Seidman et al., 2005) as compared with normal control females.

Despite the documented differences between those with ADHD and normal Controls in neurocognitive functioning, a number of confounding issues limit the generalisability of the findings and suggest caution be used when interpreting the results as indicative of specific deficits in ADHD samples. Often the issue of medication status (i.e., on or off stimulants) has not adequately been addressed (e.g., Seidman et al., 1997), IQ tends to inflate group differences found, and comorbidity of samples confuse the picture significantly. Indeed, both Rucklidge and Tannock (2002) and Purvis and Tannock (2000) suggested that many cognitive deficits attributed to ADHD, such as poor inhibition and impaired working memory, may be better attributed to the presence of reading difficulties in the ADHD samples. Other comorbidities that have been considered as influencing neurocognitive functioning of ADHD samples include Tourette's (Schuerholz, Singer, \& Denckla, 1997), and disruptive behavioural disorders and internalizing disorders (Hinshaw et al., 2002; Newcorn et al., 2001). In general, it appears that the more comorbid disorders, the more impairment found, although anxiety can decrease levels of impulsivity (Newcorn et al., 2001). 


\section{J. Rucklidge}

This current study sought to replicate and extend previous research by measuring performance across a variety of neurocognitive functions including working memory, processing speed, naming speed, inhibitory control, set-shifting and interference in a Southern Hemisphere sample. Four groups were compared: ADHD males, ADHD females, normal Control males and normal Control females. Contrary to many studies using preteen samples, this sample consisted of teenagers aged 13 to 17 years. Due to the confounding problems associated with comorbidity, comorbid status was also assessed as well as reading abilities in order to control for the effect these additional diagnoses may have on the results. Further, all ADHD participants on stimulants were tested off medication in order to mitigate the impact stimulants have on cognitive functioning. It was expected that the ADHD groups, regardless of gender, would show more impairment on neurocognitive functioning and that very few gender differences would be noted.

\section{Method}

\section{Participants}

A total of 114 adolescent youth, aged 13 to 17 years participated, 30 male Controls, 35 female Controls, 24 male ADHD, and 25 female ADHD. The ADHD group was referred through a specialized service that assesses and treats youth with moderate to severe psychiatric disorders. For participants referred from this source, a clinical psychologist evaluated the presence or absence of a current diagnosis of ADHD based on a clinical diagnostic protocol and standardised parent and teacher behaviour rating scales (described below). The Control group was recruited through advertising at local schools and other community resources, and received the same clinical evaluation as the ADHD group. The overall sample was predominantly Caucasian and of European descent (90.8\% controls, $89.8 \%$ ADHD), with the remaining being of Maori descent. 


\section{Assessments and Measures}

Diagnostic protocol for ADHD and other psychiatric disorders. The Schedule for Affective Disorders and Schizophrenia for School-Age Children-Present and Lifetime Version (KSADS-PL; Kaufman, 1997) was used to assess Diagnostic and Statistical Manual of Mental Disorders (DSM) diagnostic concepts specific to youth. The long versions of the Conners' Rating Scales-Revised (CRS-R; Conners, 1997) were also used to assess ADHD. This instrument provides separate rating forms for parents, teachers and adolescents. The parent rating involves an 80-item scale, including measures of: oppositional behaviours, hyperactivity, indices of ADHD, and cognitive problems. The 87-item adolescent self-report assesses the same areas as the parental scale, with the inclusion of anger control problems. The teacher scales consist of 59-items, which provide measures of academic, social and emotional behaviours in the classroom.

To be included in the ADHD group, a participant would have met each of the following criteria: (a) DSM-IV-TR diagnostic criteria for ADHD based on the clinician summary of the K-SADS-PL parent and adolescent interview; parental report information related to the presence versus absence of externalising symptoms would supercede the adolescent report in the event of a discrepancy; (b) a T-score $\geq 65$ on at least one of the ADHD subscales of the CRS-R parent form and, where available (not all participants attended school regularly), teacher form to ensure pervasiveness of symptoms across settings; and (c) evidence of ADHD symptoms prior to the age of seven established either through a past diagnosis of ADHD or, among new cases, through parental report and past school report cards. According to this diagnostic protocol, $11(45.8 \%)$ of the ADHD males and $10(40 \%)$ of the ADHD females were identified as Combined Type, $2(8.3 \%)$ of the ADHD males and $2(8 \%)$ of the ADHD females as Predominantly Hyperactive/Impulsive Type, and 11 (45.8\%) of the ADHD males and $13(53 \%)$ of the ADHD females as Predominantly Inattentive Type. 


\section{J. Rucklidge}

To be included in the Control group, an adolescent would have failed to meet ADHD criteria according to the K-SADS-PL and had T scores $<65$ on the ADHD subscales of the parent and teacher form of the CRS-R.

Exclusion criteria for all groups. Children were excluded from analyses if they had: (a) an estimated IQ below 75 using the Block Design and Vocabulary subtests of the Wechsler Adult Intelligence Scale-III (WAIS-III; Wechsler, 1997) or the Wechsler Intelligence Scale for Children-III (WISC-III; Wechsler, 1991), a combination of subtests commonly used to estimate full scale IQ (Sattler, 2001), or (b) uncorrected problems in vision or hearing, serious medical problems, such as epilepsy or cerebral palsy, or serious psychopathology, such as psychosis, that would preclude a current differential diagnosis of ADHD. Five participants were excluded due to low IQ (4 ADHD, 1 NC).

Parents of children in the ADHD group who were taking psycho-stimulant medication (i.e., methylphenidate; $n=31$ or $63.3 \%$ ) were asked not to give their children this medication on the morning of testing as stimulant medications can enhance task performance (Trommer, Hoeppner, \& Zecker, 1991; Wilkison, Kircher, McMahon, \& Sloane, 1995). As methylphenidate has an approximate half-life of 4.5 hours (Shader et al., 1999), a 24-hour elimination period should have ensured that the majority of the active ingredient had been eliminated prior to testing. Nine (18.4\%) of the ADHD group and two $(3.1 \%)$ of the controls were taking a medication other than a stimulant (paroxetine, clonidine, fluoxetine, citalopram). These other medications were not discontinued.

\section{Measures of demographic variables and potential covariates}

The New Zealand Socioeconomic Index of Occupational Status (NZSEI) was used to establish an Socio Economic Status (SES) ranking for each of the participants. The NZSEI 
was designed by Davis, McLeod, Ransom and Ongley (1997) as a measure of SES, and is based upon two widely used scales of SES in New Zealand, the Health and Equity (HEQ) Index and the Elley-Irving Occupational Scale. The NZSEI scores which range between 10 and 100 (with higher scores indicating higher SES), is based on 1991 New Zealand census data. Each participant in the present study was ranked according to the NZSEI based on his/her parent's occupation.

Assessment of comorbid symptomatology. The K-SADS-PL (Kaufman et al., 1997) was used to assess the presence/absence of most Axis I disorders. In the present research, an aggregate continuous variable that represented the presence of these disorders was derived by adding together the most common diagnoses and dividing by the number of diagnoses included. Table 1 shows the frequency of the most common diagnoses across the four groups.

\section{[t]Table 1 near here[/t]}

Assessment of reading abilities. The Wechsler Individual Achievement Test, Second Edition (WIAT-II: The Psychological Corporation, 2001) was used to measure word reading (assesses pre-reading and decoding skills), spelling (evaluates the ability to spell), and psuedoword decoding (assesses child's ability to apply phonetic decoding skills). The test is suitable for individuals aged 4 years to 85 years $\{$ Wechsler, $2001 \# 480\}$. An average of these three tests was calculated and considered as a covariate in the analyses given the documented impact Corporation, 2001) reading abilities can have on neurocognitive functioning (Rucklidge \& Tannock, 2002). This average was also used for categorical purposes as well: those below 85 on this conglomerate were classified as $\mathrm{RD}$, and those above were classified as nonRD. This allowed for documentation of the percent in each group with significant impairment in reading. 


\section{J. Rucklidge}

\section{Dependent measures}

Naming and Processing Speed. 1) Five tests of Rapid Automatized Naming (RAN) were selected to assess for rapid naming: Letters, Numbers, Colors, Objects, and Colors/Numbers/Letters (Denckla \& Rudel, 1974). This test was selected because of its association with more effortful semantic naming and its established association with ADHD (Carte et al., 1996; Semrud-Clikeman, Guy, Griffin, \& Hynd, 2000). RAN-Letters consists of five lowercase letters repeated ten times in random sequence, yielding 50 stimuli presented in five rows of ten items on a chart. RAN-Numbers consists of 5 digits, RAN-Colors consists of five colours and RAN-Objects consists of five objects presented in the same way as RANLetters. Finally, RAN-Colors/Letters/Numbers consists of 50 alternating colours, letters and numbers. Total times (in seconds) to name all stimulus items on each chart were the dependent variables. Number stated correctly, number of omissions, additions, deletions, and errors were also assessed as control variables, although errors tend to be infrequent in this population. 2) The Processing Speed Index, consisting of Symbol Search and Coding subtests, of the WISC-III/WAIS-III, was used to assess speed of information processing.

Memory. 1) The short form of the Wide Range Assessment of Memory and Learning (WRAML: Sheslow, 1990) was administered. This test is normed for children 5 to 17 years of age and the short-form consists of four subtests: Picture Memory, Design Memory, Verbal Learning and Story Memory. Finger Windows, a measure of spatial span, was also administered in order to obtain a Visual Memory Index. Standard scores are provided and the higher the score, the better the memory. Reliability scores range from .70 to .94 for subtest scores and .88 to .97 for index scores. Validity has been confirmed with comparisons to other memory scales. 2) Verbal working memory was assessed using the WISC-III/WAIS-III Freedom from Distractibility/Working Memory Index. This index consists of Arithmetic and 
Digit Span in the case of the WISC-III and these two subtests as well as Letter-Number Sequencing for the WAIS-III.

Response inhibition (protection from interference). The Stroop Color and Word Test (Golden, 1978) was administered, yielding four dependent measures, namely, number of colour words (red/blue/green) named in 45 seconds, number of colours (red/blue/green) named in 45 seconds, number of colour names that are printed in a discordant colour word named within 45 seconds (e.g., when the word "red" is written in green ink, the subject must respond with "green"), and an interference estimate that measures the ability to suppress a habitual response in favour of an unusual one, taking into account overall speed of naming. T scores are provided by Golden (1978) for the age groups studied.

Planning and Set-shifting. The computerised version of the Wisconsin Card Sorting Task (WCST: Heaton, Chelune, Tally, Kay, \& Curtis, 1993), a test designed for individuals aged 6.5 to 89 years was administered. It has been considered a measure of executive function, requiring the ability to develop and maintain an appropriate problem-solving strategy across changing stimulus conditions in order to achieve a future goal. Not only does it provide objective scores of overall success, but also for specific sources of difficulty on the task. Normative indices used as dependent variables in this study included number of perseverative errors, percent conceptual level responses, and number of categories achieved, and (Au: is it ok to add "and" here?) were chosen as global indices tapping into executive abilities. There is some evidence that the test is sensitive (although not necessarily specific) to frontal lobe dysfunction in children (Heaton et al., 1993). Test-retest is only moderate (.37 - .72; Heaton et al., 1993) which is likely because once an individual has determined the categories' sort and shift principle, it is no longer a test of problem-solving ability. 
Visual scanning and cognitive flexibility. The Color Trails Test (CCT) was designed to minimise the influence of language and learning disabilities, and covers the full child to adult age range although norms are only available for individuals over 18 years of age (D'Elia, 1996). Part I is similar to Trails A except that the odd-numbered circles have a pink background and the even-numbered circles have a yellow background. Part 2 shows all the numbers from 1 to 25 twice, one with a pink background and one with a yellow background. Scoring is expressed in terms of time in seconds to complete Part A and Part B of the test. An interference ratio (ratio of Part 2 minus 1 over Part 1 time scores) is also calculated. Colour errors, number errors, near-misses and prompts are also recorded; however, these errors are rare in a normative population. Williams et al. (1995) found good correlations between the CCT and the TMT, good discriminant validity between normal control children and children with altered neuropsychological functions, and appropriate age-progression of scores.

Response inhibition. The Conners' Continuous Performance Test (CPT-II: Connors, 2000) was used as a measure of complex cognitive functioning, including attention, visual-motor speed, visual-motor integration, hyperactivity and impulsivity. The task takes 14 minutes to complete and in brief, the child is required to respond to the computer screen by pressing a space bar for every letter except the letter "X". The computer generates an output that includes standardised scores of number of omissions (believed to be related to inattention), number of commissions (believed to be a measure of impulsivity), reaction time, and variability of reaction time. These scores were used as dependent variables due to their hypothesized association with ADHD functioning. A confidence index is also provided. While the CPT has been found to distinguish between children with ADHD and normal Controls, particularly the signal detection measures (Epstein et al., 2003), it has not been 
successful at reliably distinguishing between children with ADHD and clinical Controls thus questioning its diagnostic validity (McGee, Clark, \& Symons, 2000). Only one study to date has investigated the validity of the CPT-II, suggesting that it showed poor correlations with external measures of inattention and hyperactivity (Weis \& Totten, 2004). Research on the reliability and validity of this measure is limited.

\section{Procedures}

The clinical interviews and testing were conducted in clinical laboratories within a department of psychology in a midsized university. Consent and assent forms were reviewed with both parents and adolescents. Questionnaire packages were sent to the adolescents' teachers with parental consent. The interviews were conducted with both the parent and adolescent separately by doctoral level registered psychologists with extensive experience in interviewing adolescents and families and trained to identify problems that may be in need of a referral. All cases were reviewed with the primary investigator and every ten interviews were videoed (approximately 10\%) and reviewed by a second rater to assess for diagnostic reliability. There was $100 \%$ agreement on the presence/absence of all Axis I disorders. The performance and adolescent self-report measures and cognitive measures were administered in the same order by psychometrists blind to the diagnostic status of the child. All participants were provided with feedback and a report.

\section{Results}

\section{Statistical Analyses}

Results were analysed using the Statistical Package for the Social Sciences-Windows Version 12. Univariate tests were performed on the dependent measures of interest. In an effort to balance Type I and Type II errors, interpretations were based on $p<.01$; however, the tables 
indicate significance at three levels: $.05, .01, .001$. Three planned comparisons were performed: 1) ADHD males were compared with male Controls; 2) ADHD females were compared with female Controls; and 3) ADHD females were compared with ADHD males. Five variables were considered as covariates: age, SES, estimated IQ, comorbidity, and reading ability. Chi-square analyses were used for group comparisons of the dichotomous variables. Effect sizes were calculated according to Cohen's (1988) effect size computation ( $d$ $=\mathrm{M}_{1}-\mathrm{M}_{2} / \sqrt{ }\left(\left(\mathrm{SD}_{1}^{2}+\mathrm{SD}_{2}^{2}\right) / 2\right)$.

\section{Preliminary Analyses}

Participant characteristics as a function of group membership. A series of preliminary analyses examined the distribution of demographic characteristics in relation to group membership. As shown in Table 2, no age or SES differences were noted as a function of group membership. The mean overall SES level of the sample was $56.30(S D=20.67)$, which is indicative of middle socio-economic status. Only the female ADHD and female Controls differed in estimated IQ, with the females with ADHD having a lower IQ than the Controls. Therefore, IQ was used as a covariate in the female comparisons. Both presence of comorbidity and average reading ability were significantly different between both the ADHD females and female Controls, and the ADHD males and the male Controls, with the ADHD groups showing more difficulties with reading and more comorbid diagnoses. Therefore, these two variables were included as covariates in the Control/ADHD comparisons. Using a cutoff of 85 on the WIAT-II average reading score, three (10\%) of the male Controls, one $(2.9 \%)$ of the female Controls, eight $(33.3 \%)$ of the male ADHD, and four $(16 \%)$ of the female ADHD showed significant impairment in reading. As expected, group differences were found between the Controls and ADHD group on the Conners Rating Scales. 


\section{[t]Table 2 near here[/t]}

\section{Processing speed and speed of naming}

Table 3 shows that the ADHD groups, regardless of gender, have significant difficulties with processing speed and naming speed. No group differences were found between the males and females with ADHD. Both males and females with ADHD were particularly slow at naming letters, colours, and objects, even after controlling for covariates, as compared with the Controls. Some group differences were eliminated when covariates were considered, in particular, the group differences on number naming and colour/number/letter naming speed. Effect sizes were large (> .8) between the ADHD groups and Controls and small (.2) between the ADHD males and ADHD females, confirming the group differences found. No group differences were found on the RAN in numbers or errors, commissions, omissions, and so on.

\section{[t]Table 3 near here[/t]}

\section{Memory}

Overall, the females with ADHD showed the most problems with memory as compared with female Controls. Indeed, after controlling for covariates, no group differences were found between the ADHD males and male Controls. The females with ADHD showed specific deficits in recall of designs, overall visual memory, verbal arithmetic abilities, and working memory abilities. Further, the ADHD females performed more poorly on tests of spatial memory (spatial span and design memory) as compared with the ADHD males, although these differences were not significant when controlling for type I error. Effect sizes were medium (.4 to .6) for those tests not reaching statistical significance between the ADHD groups and controls, but large (.8) between the ADHD groups and Controls where group differences were noted. Effect sizes were generally larger between the females with ADHD 
and female Controls as compared with effect sizes between the ADHD males and male Controls.

\section{[t]Table 4 near here[/t]}

\section{Executive functioning}

Both the females and males with ADHD showed poorer performance on the Stroop Word and Color naming, and some differences were noted on the CPT-II. In particular, the ADHD males were more variable in their responses than the Control males and the ADHD females made more omission errors as compared with the Controls. Further, the ADHD males were more impaired in Stroop Word, were more variable in their responses on the CPT-II and were more likely to be classified as ADHD according to the confidence index as compared with the females with ADHD. There were no group differences on the Wisconsin Card Sorting Task, interference scores on both the Stroop and Trails, and while a few group differences emerged on Color Trails I and II, these disappeared when covariates were included. Effect sizes confirmed the lack of group differences found in that they were small $(<.2)$ for the WCST between the ADHD groups and Controls and large $(>.8)$ where group differences were found on Stroop and CPT-II.

\section{[t]Table 5 near here[/t]}

\section{Other analyses}

Given the group differences noted, the univariate analyses were rerun using gender (male/female) and ADHD (present/absent) as independent variables in order to assess groupby-gender interactions. One significant interaction was found on Design Memory of the WRAML: $F(1,107)=4.828, p<.05$. This interaction suggests the combination of having 
ADHD and being female influences impaired performance on design memory; however, caution should be exerted interpreting this result as it is the only significant difference among 33 analyses, a finding easily explained by chance alone.

Those ADHD children on medication were compared with those off medication and no group differences were found in neurocognitive functioning, suggesting that medications cannot account for the effects found.

\section{Discussion}

This study used a broad range of tests assessing across multiple cognitive areas, including memory, processing speed, naming speed, inhibition, and interference, to document the neuropsychological performances of both males and females with ADHD as compared with Controls in addition to identifying gender differences within the ADHD sample. This current study supports a growing body of literature showing that females with ADHD are as impaired as males with ADHD in most areas of neuropsychological functioning; indeed, few gender differences were noted within the ADHD sample. Specifically, males and females with ADHD showed comparable functioning on tasks of naming speed, processing speed, working memory, set-shifting, interference, and number of omissions and commissions in a continuous performance task. Females with ADHD were slightly more impaired in spatial span and spatial memory, whereas males with ADHD were more variable in their performance on the test of continuous performance and were marginally slower at naming colour words than ADHD females.

In comparison to the normal Controls, both ADHD groups showed impaired memory (not evident with the males after controlling for reading and comorbidity status), slowed naming speed, and slowed processing abilities. There were no group differences on set-shifting (WCST) and while group differences were found on the continuous performance task and 
colour trails, the differences weakened when controlling for reading ability, IQ, and other comorbid disorders. The overall lack of gender by ADHD interactions suggest that the risk for ADHD-associated impairments is similarly elevated in both males and females with ADHD. Effect sizes generally confirmed the group differences found and indicated large effect sizes between the ADHD groups and the Controls and small effect sizes between the ADHD males and ADHD females. This study supports other research in finding very few gender differences in an ADHD sample in intellectual functioning and overall executive functioning in an ADHD sample (Arcia \& Conners, 1998; Seidman et al., 2005), and those of other studies showing impaired neurocognitive functioning in ADHD females as compared with controls (Hinshaw, 2002; Seidman et al., 2005). While most studies have used North American and European samples, these deficits are now noted in a New Zealand sample.

The results concerning memory abilities in the sample are intriguing. While some differences were noted in overall memory abilities of the ADHD sample, particularly the females with ADHD, controlling for other factors limited many group differences, suggesting that some of the documented problems in memory abilities in ADHD samples are better attributable to comorbid conditions or poorer overall cognitive abilities than a specific deficit related to ADHD; findings found in other ADHD samples as well (Kaplan, Dewey, Crawford, \& Fisher, 1998). However, even after controlling for these other variables, females with ADHD continued to show impaired verbal working memory and impaired spatial memory, supporting a recent meta-analysis confirming that working memory problems are consistently found in ADHD samples (Martinussen et al., 2005), but implying that the deficits may be stronger in females than males. It is also possible that the fact that the normal Control females performed particularly well on the memory task inflated the group differences between the female Controls and females with ADHD. 
Consistent with other studies, both males and females showed some impaired performance on the Stroop. However, no group difference emerged on the measure of interference (which controls for overall slowed naming speed), a finding consistent with more recent literature (van Mourik, Oosterlaan, \& Sergeant, 2005). A more reliable finding, although influenced by reading ability in the female ADHD sample, was impaired rapid verbal naming, particularly colour and object naming, consistent with other researchers (Nigg, 2001; Rucklidge \& Tannock, 2002). However, impairment is unlikely to be specific to ADHD as problems in rapid naming are cardinal features of RD samples as well (Purvis \& Tannock, 2000).

No group differences were found on the Wisconsin Card Sorting Task, contrary to the findings of problems with set-shifting in the sample reported by Seidman and colleagues (2005) and Lawrence and colleagues (2004). However, these results support previous reports on ADHD children who have not identified deficits on the WCST (Willcutt et al., 2001). The fact that the computerized version was administered in the current study could have influenced the overall results in that the computerised version is slower in administration and therefore allows for more time to consider responses, and there is less room for experimenter error, a significant problem when administered manually. It is also possible that the lack of group differences found in set-shifting abilities in addition to the weak findings on visual mental flexibility as measured by Color Trails are due to an older sample being tested and that by the teenage years, some functioning that was impaired when younger, has caught up developmentally.

This study found that the males with ADHD showed more variability in responses to the CPT-II compared to the females with ADHD and this group difference likely also led to higher confidence indices for the ADHD males. Indeed, while the confidence index was $66 \%$ for the ADHD males, it was only $47 \%$ for the ADHD females, a significant group difference, although not too meaningful clinically given that this index indicates that many of the ADHD 
males and females were performing relatively well on this task. These results support a growing body of literature suggesting that the CPT-II does not aid clinicians with ADHD diagnoses (McGee et al., 2000; Nichols \& Waschbusch, 2004), and may be more useful with the combined subtype (Collings, 2003). Further, this current study suggests that the test may be more useful with ADHD males. In other words, results on this test, if used in a clinical capacity, should be interpreted with great caution, particularly normal results in females being considered for an ADHD diagnosis. Newcorn et al. (2001) also found less impairment of their ADHD girls on the CPT, finding that they made less impulsivity errors (commission errors) than the ADHD boys.

While ADHD subtyping was not considered in these analyses, the fact that there was an equal distribution of the three subtypes across males and females meant that subtype unlikely influenced the results. Further, there were no group differences between males and females with ADHD on the Conners subscales assessing for ADHD inattention and hyperactivity/impulsivity, thereby suggesting that the ADHD groups were showing similar levels of severity in ADHD symptomatology.

It should be acknowledged that the sample size was small and therefore limited the number of analyses that could be performed, including comparisons within subtypes of ADHD. As with many studies on ADHD, the sample used in this study was a clinically referred sample and likely more impaired than a community sample. Generalisability across samples needs to be made with caution. Many tests were performed and therefore fatigue, particularly if it affected one group more than another, could explain some noted group differences. While stimulant medications were not taken on the day of testing, without assays, it cannot be concluded that the children were free of the active ingredients in these medications. 


\section{Clinical implications}

Although there are fewer females being diagnosed with ADHD, the evidence is now clearly showing that females with ADHD are as impaired in neurocognitive functioning as their male counterparts. While deficits are found in all studies investigating neurocognitive functioning of ADHD populations, the fact that inconsistencies are noted across the studies in which tests show deficits implies that variability in neurocognitive performance in the ADHD population may be the norm rather than the exception, making the task of finding which brain regions are implicitly involved in ADHD symptomatology all the more arduous. In practice, these findings of variable problems in ADHD participants urge clinicians to routinely test for cognitive strengths and weaknesses in all their ADHD clients in order to tailor interventions to the specific client profile. In other words, if, for example, working memory is a problem, then strategies that target such difficulties would be included, such as lists, mnemonics, and relying on other cognitive strengths. Other children may show specific deficits in processing speed, in which case, they may benefit from additional time in tests and taping lessons. At this point in time, while comorbid profiles can suggest specific neurocognitive deficits, particularly presence of reading problems, assumptions cannot be made about which deficits will be present in which individual with ADHD.

\section{Author Note}

This research was partially supported by Research Grant U6497 from the University of Canterbury. The author extends gratitude to Tamara Clancy, Paula Bateup, Fiona Prest, James Anderson and Nicola Ward for their assistance with their data collection.

\section{References}

Arcia, E., \& Conners, C. K. (1998). Gender differences in ADHD? Journal of Developmental and Behavioral Pediatrics, 19, 77-83. 
American Psychiatric Association (2000). Diagnostic and statistical manual of mental disorders: DSM-IV-TR (4th ed., text revision). Washington, DC: Author.

Barkley, R. A. (1997). Behavioral inhibition, sustained attention, and executive functions: Constructing a unifying theory of ADHD. Psychological Bulletin, 121, 65-94.

Biederman, J., Faraone, S., Monuteaux, M., Bober, M., \& Cadogen, E. (2004). Gender effects on Attention-Deficit/Hyperactivity Disorder in adults, revisited. Biological Psychiatry, 55, $692-700$.

Carte, E. T., Nigg, J. T., \& Hinshaw, S. P. (1996). Neuropsychological functioning, motor speed, and language processing in boys with and without ADHD. Journal of Abnormal Child Psychology, 24, 481-498.

Castellanos, F. X., Marvasti, F. F., Ducharme, J. L., Walter, J. M., Israel, M. E., Krain, A., et al. (2000). Executive functioning oculomotor tasks in girls with ADHD. Journal of the American Academy of Child and Adolescent Psychiatry, 39, 644-650.

Cohen, J. (1988). Statistical power analysis for the behavioral sciences (2nd ed.). Hillsdale, NJ: Lawrence Erlbaum Associates.

Collings, R. D. (2003). Differences between ADHD inattentive and combined types on the CPT. Journal of Psychopathology and Behavioral Assessment, 25, 177-189.

Conners, C. K. (1997). Conners' Rating Scales-Revised: Technical manual. New York: MultiHealth Systems Inc.

Connors, C. K. (2000). Connors Continuous Performance Test II: Technical Guide. Toronto, Cananda: Multi-Health Systems.

Davis, P., McLeod, K., \& Ransom, M. (1997). The New Zealand Socioeconomic Index of Occupational Status (NZSEI) (Research Rep. No. 2). Wellington: Statistics New Zealand.

D'Elia, L., Satz, P., Uchiyama, C., \& White, T. (1996). Color Trails Test: Professional Manual. Odessa, FL: Psychological Assessment Resources. 
Denckla, M. B., \& Rudel, R. G. (1974). "Rapid automized naming" of pictured objects, colors, letters, and numbers by normal children. Cortex, 10, 186-202.

Epstein, J. N., Erkanli, A., Conners, K., Klaric, J., Costello, J., \& Angold, A. (2003). Relations between the Continuous Performance Test performance measures and ADHD behaviors. Journal of Abnormal Child Psychology, 31, 543-554.

Gaub, M., \& Carlson, C. L. (1997). Gender differences in ADHD: A meta-analysis and critical review. Journal of the American Academy of Child and Adolescent Psychiatry, 36, 1036-1045.

Gershon, J. (2002). A meta-analytic review of gender differences in ADHD. Journal of Attention Disorders, 5, 143-154.

Golden, C. J. (1978). Stroop Color and Word Test: A manual for clinical and experimental uses. Wood Dale, Il: Stoelting Company.

Heaton, R. K., Chelune, G. J., Tally, J. L., Kay, G. G., \& Curtis, G. (1993). Wisconsin Card Sorting Test (WCST) manual revised and expanded. Odessa, FL: Psychological Assessment Resources, Inc.

Hinshaw, S. P. (2002). Preadolescent girls with Attention-Deficit/Hyperactivity Disorder: I. Background characteristics, comorbidity, cognitive and social functioning, and parenting practices. Journal of Consulting and Clinical Psychology, 70(5), 1086-1098.

Hinshaw, S. P., Carte, E. T., Sami, N., Treuting, J. J., \& Zupan, B. A. (2002). Preadolescent girls with Attention-Deficit/Hyperactivity Disorder: II. Neuropsychological performance in relation to subtypes and individual classification. Journal of Consulting and Clinical Psychology, 70(5), 1099-1111.

Kaplan, B. J., Dewey, D., Crawford, S. G., \& Fisher, G. C. (1998). Deficits in long-term memory are not characteristic of ADHD. Journal of Clinical and Experimental Neuropsychology, 20, 518-528. 
Kaufman, J., Birmaher, B., Brent, D., Rao, U., Flynn, C., Moreci, P., et al. (1997). Schedule for Affective Disorders and Schizophrenia for School-age Children-present and Lifetime Version (K-SADS-PL): Initial reliability and validity data. Journal of the American Academy of Child and Adolescent Psychiatry, 36, 980-987.

Lawrence, V., Houghton, S., Douglas, G., Durkin, K., Whiting, K., \& Tannock, R. (2004). Executive function and ADHD: A comparison of children's performance during neuropsychological testing and real-world activities. Journal of Attention Disorders, 7, 137-149.

Martinussen, R., Hayden, J., Hogg-Johnson, S., \& Tannock, R. (2005). A meta-analysis of working memory impairments in children with Attention-Deficit/Hyperactivity Disorder. Journal of the American Academy of Child and Adolescent Psychiatry, 44, 377-384.

McGee, R. A., Clark, S. E., \& Symons, D. K. (2000). Does the Conners' Continuous Performance Test aid in ADHD diagnosis? Journal of Abnormal Child Psychology, 28, $415-424$.

Nada-Raja, S., Lagley, J. D., McGee, R., Williams, S. M., Begg, D. J., \& Reeder, A. I. (1997). Inattentive and hyperactivity behaviors and driving offences in adolescence. Journal of the American Academy of Child and Adolescent Psychiatry, 36, 515-522.

Newcorn, J., Halperin, J. M., Jensen, P., Abikoff, H. B., Arnold, L. E., Cantwell, D. P., et al. (2001). Symptom profiles in children with ADHD: Effects of comorbidity and gender. Journal of the American Academy of Child and Adolescent Psychiatry, 40, 137-146.

Nichols, S. L., \& Waschbusch, D. A. (2004). A review of the validity of laboratory cognitive tasks used to assess symptoms of ADHD. Child Psychiatry and Human Development, 34, 297-315.

Nigg, J. T. (2001). Is ADHD a disinhibitory disorder? Psychological Bulletin, 127, 571-598. 
Purvis, K. L., \& Tannock, R. (2000). Phonological processing, not inhibitory control, differentiates ADHD and reading disability. Journal of the American Academy of Child and Adolescent Psychiatry, 39, 485-494.

Rucklidge, J. J., \& Tannock, R. (2001). Psychiatric, psychosocial, and cognitive functioning of female adolescents with ADHD. Journal of the American Academy of Child and Adolescent Psychiatry, 40, 530-540.

Rucklidge, J. J., \& Tannock, R. (2002). Neuropsychological profiles of adolescents with ADHD: Effects of reading difficulties and gender. Journal of Child Psychology and Psychiatry, 43, 988-1003.

Sattler, J. M. (2001). Assessment of children. Cognitive applications (4th ed.). San Diego: Jerome M. Sattler, Publisher, Inc.

Schuerholz, L. J., Singer, H., \& Denckla, M. B. (1997). Gender study of neuropsychological and neuromotor function in children with Tourette Syndrome with and without AttentionDeficit/Hyperactivity Disorder. Journal of Child Neurology, 13, 277-282.

Schulz, K. P., Fan, J., Tang, C. Y., Newcorn, J. H., Buchsbaum, M. S., Cheung, A. M., et al. (2004). Response inhibition in adolescents diagnosed with attention deficit hyperactivity disorder during childhood: An event-related fMRI study. American Journal of Psychiatry, $161,1650-1657$.

Seidman, L. J., Biederman, J., Faraone, S. V., Weber, W., Mennin, D., \& Jones, J. (1997). A pilot study of neuropsychological function in girls with ADHD. Journal of the American Academy of Child and Adolescent Psychiatry, 36, 366-373.

Seidman, L. J., Biederman, J., Monuteaux, M. C., Valera, E., Doyle, A. E., \& Faraone, S. V. (2005). Impact of gender and age on executive functioning: Do girls and boys with and without Attention Deficit Hyperactivity Disorder differ neuropsychologically in preteen and teenage years? Developmental Neuropsychology, 27, 79-105. 
Semrud-Clikeman, M., Guy, K., Griffin, J. D., \& Hynd, G. W. (2000). Rapid naming deficit in children and adolescents with reading disabilities and attention deficit hyperactivity disorder. Brain and Language, 74, 70-83.

Shader, R. I., Harmatz, J. S., Oesterheld, J. R., Parmelee, D. X., Sallee, F. R., \& Greenblatt, D. J. (1999). Population pharmacokinetics of methylphenidate in children with attentiondeficit/hyperactivity disorder. Journal of Clinical Pharmacology, 39, 775-785.

Sheslow, D., \& Adams, W. (1990). Wide Range Assessment of Memory and Learning Administration Manual. Wilmington, DE: Jastak (Au: please include town .......:DE).

Slaats-Willemse, D., Swaab-Barneveld, H., De Sonneville, L., Van Der Meulen, E., \& Buitelaar, J. (2003). Deficient response inhibition as a cognitive endophenotype of ADHD. Journal of the American Academy of Child and Adolescent Psychiatry, 42, 1242-1248.

Tannock, R. (1998). Attention deficit hyperactivity disorder: Advances in cognitive, neurobiological, and genetic research. Journal of Child Psychology and Psychiatry and Allied Disciplines, 39, 65-99.

Tannock, R., Martinussen, R., \& Fritjers, J. (2000). Naming speed performance and stimulant effects indicate effortful, semantic processing deficits in attention-deficit/hyperacitivity disorder. Journal of the American Academy of Child and Adolescent Psychiatry, 28, 237252.

The Psychological Corporation, (2001). Wechsler Individual Achievement Test, Second Edition. San Antonio, TX: Harcourt Assessment Company. (Au: please include town.

\section{Also does the "P" above belong?)}

Toplak, M. E., Rucklidge, J. J., Hetherington, R., John, S. C. F., \& Tannock, R. (2003). Time perception deficits in attention-deficit/hyperactivity disorder and comorbid reading difficulties in child and adolescent samples. Journal of Child Psychology and Psychiatry, $44,888-903$. 
Trommer, B. L., Hoeppner, J. B., \& Zecker, S. G. (1991). The go-no go test in attention deficit disorder is sensitive to methylphenidate. Journal of Child Neurology, 6 (Suppl.), S126-S129.

van Mourik, R., Oosterlaan, J., \& Sergeant, J. A. (2005). The Stroop revisited: A metaanalysis of interference control in AD/HD. Journal of Child Psychology and Psychiatry, $46,150-165$.

Wechsler, D. (1991). Manual for the WISC-III. New York, NY: Psychological Corporation. Wechsler, D. (1997). Manual for the WAIS-III. New York, NY: Psychological Corporation.

Weis, R., \& Totten, S. J. (2004). Ecological validity of the Conners' Continuous Performance Test II in a school-based sample. Journal of Psychoeducational Assessment, 22, 47-61.

West, J., Houghton, S., Douglas, G., \& Whiting, K. (2002). Response inhibition, memory and attention in boys with Attention-Deficit/Hyperactivity Disorder. Educational Psychology, 22(5), 533-551.

Wilkison, P. C., Kircher, J. C., McMahon, W. H., \& Sloane, H. N. (1995). Effects of methylphenidate on reward strength in boys with attention-deficit/hyperactivity disorder. Journal of the American Academy of Child and Adolescent Psychiatry, 34, 897-901.

Willcutt, E. G., Pennington, B. F., Boada, R., Ogline, J. S., Tunick, A., Chhabildas, N. A., et al. (2001). A comparison of the cognitive deficits in reading disability and AttentionDeficit/Hyperactivity Disorder. Journal of Abnormal Psychology, 110, 157-172.

Williams, J., Rickert, V., Hogan, J., Zolten, A. J., Satz, P., D'Elia, L. F., et al. (1995). Children's Color Trails. Archives of Clinical Neuropsychology, 10, 211-223. 